\title{
Two adjacent nuclear factor-binding domains activate expression from the human PRNP promoter
}

\author{
Michael J Taheny, Nerik Izkhakov, Alexander A Vostrov and \\ Wolfgang W Quitschke*
}

Address: Department of Psychiatry and Behavioral Science, State University of New York at Stony Brook, Stony Brook, NY 11794-8101, USA Email: Michael J Taheny - m_taheny@hotmail.com; Nerik Izkhakov - nizkhakov@gmail.com; Alexander A Vostrov - Alvostrov@gmail.com; Wolfgang W Quitschke* - wquitschke@notes.cc.sunysb.edu

* Corresponding author

Published: 9 September 2009

BMC Research Notes 2009, 2:178 doi:10.1 186/1756-0500-2-178
Received: 25 June 2009

Accepted: 9 September 2009

This article is available from: http://www.biomedcentral.com/1756-0500/2//78

(C) 2009 Quitschke et al; licensee BioMed Central Ltd.

This is an open access article distributed under the terms of the Creative Commons Attribution License (http://creativecommons.org/licenses/by/2.0), which permits unrestricted use, distribution, and reproduction in any medium, provided the original work is properly cited.

\begin{abstract}
Background: The transmissible spongiform encephalopathies (TSEs) comprise a group of fatal degenerative neurological diseases in humans and other mammals. After infection, the cellular prion protein isoform $\mathrm{PrPC}$ is converted to the pathological PrPSC scrapie isoform. The continued conversion of $\mathrm{PrPC}$ to $\mathrm{PrPSC}$ requires de novo endogenous PrP synthesis for disease progression. The human prion protein gene (PRNP) promoter was therefore investigated to identify regulatory elements that could serve as targets for therapeutic intervention.

Findings: The human prion protein gene (PRNP) promoter from position -1593 to +134 relative to the putative transcriptional start site $(+I)$ was analyzed by transient transfection in HeLa cells. Deletions from the 5 ' end between positions -1593 and -232 yielded little change in activity. A further $5^{\prime}$ deletion at position -90 resulted in a decline in activity to a level of about $30 \%$ of the fulllength value. DNase I footprinting of the region between positions -259 and +2 identified two adjacent protected domains designated as prpA (-1 I6 to -143) and prpB (-I47 to - I86). Internal deletions combined with mobility shift electrophoresis and methylation interference assays indicated the presence of sequence specific nuclear factor complexes that bind to the prpA and PrPB domains and activate expression from the human PRNP promoter in an additive fashion.
\end{abstract}

Conclusion: Results from transient transfection, DNase I footprinting, mobility shift electrophoresis, and methylation interference experiments suggest that two DNase I protected domains designated as prpA and prpB are binding sites for as yet unidentified regulatory factors that independently activate expression from the PRNP promoter.

\section{Background}

The TSEs comprise a group of fatal degenerative neurological diseases in mammals [1]. After infection, the endogenous cellular prion protein isoform $\mathrm{PrPC}^{\mathrm{C}}$ is converted to the pathological PrPSC scrapie isoform. The continued conversion of $\mathrm{PrPC}^{\mathrm{C}}$ to $\mathrm{PrPSC}^{\mathrm{SC}}$ requires de novo $\mathrm{PrP}$ synthesis
[2-4]. These observations suggest that a reduction in PRNP expression could ameliorate the progression of TSEs.

The human PRNP contains two exons with the ORF located on exon 2 and the $5^{\prime}$ untranslated region of the mRNA on exon 1 [5]. The PRNP is expressed at varying 
levels in many tissues and it is developmentally regulated $[6,7]$. PRNP expression was reported to be upregulated by copper ions, NGF, hypoxia, and hypoglycemia [8-12]. The PRNP promoter region has been characterized for the bovine, murine, rat, marsupial, and human genes [13-18]. It lacks a TATA box but has a high GC content and contains recognition sequences for numerous transcription factors [18]. Polymorphisms in the human and bovine promoters have been linked to increased susceptibility to prion diseases $[19,20]$. The human PRNP promoter was here further analyzed to identify additional regulatory mechanisms.

\section{Results and Discussion}

\section{Transfection with PRNP promoter 5' deletions}

The full-length PRNP promoter and its 5' deletions (Fig. $1 \mathrm{~A}$ and $1 \mathrm{~B}$ ) were transiently transfected into HeLa cells. Expression from the promoter remained largely unchanged at full-length levels to position -232 (Bpm I) relative to the transcriptional start site $(+1)[21]$ (Fig. 1C). A further deletion at position -90 (Afe I) resulted in a significant decline in activity to about $30 \%$ of the full-length value ( $\mathrm{p}<0.0001)$. Additional statistically highly significant decreases in activity were observed at position -17 (Mfe I, p < 0.0001) and +101 (Nar I, p < 0.0001) [Fig. 1C]. These results indicated the presence of a significant activating domain between position -232 and -90. Additional domains that are essential for PRNP expression are likely to exist downstream from position -45 . However, these were not further examined here since they are close to the transcriptional start site and presumably involve factors associated with transcriptional initiation.

Other similar transfection studies with PRNP promoter deletions have yielded alternative results $[17,18]$. In one report, variable promoter expression from deletions between positions -1543 and -284 was observed, suggesting the presence of positive and negative regulatory factors interacting with that region [17]. The reasons for these divergent observations may be related to differences in cell types, reporter systems, and constructs used.

\section{DNase I footprinting}

To determine if the region between positions -232 and -90 contains nuclear factor binding sites, a fragment extending from position -259 to +2 was generated by PCR and $5^{\prime}$ end-labeled on the coding strand. The fragment was incubated with HeLa cell nuclear extract and partially digested with DNase I. Two prominent adjacent footprints designated as prpA (-116 to -143$)$ and prpB (-147 and -186$)$ were identified (Fig. 2).

\section{Mobility shift electrophoresis and methylation interference}

Mobility shift electrophoresis with double stranded oligonucleotide prpA resulted in three binding complexes des-

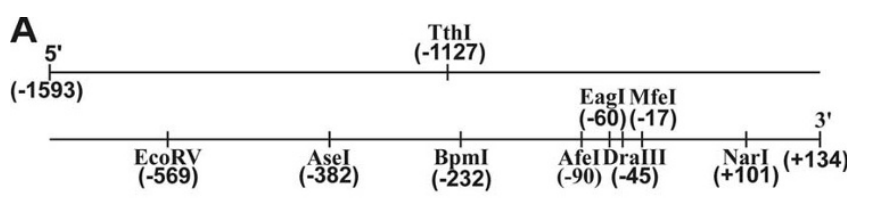

B

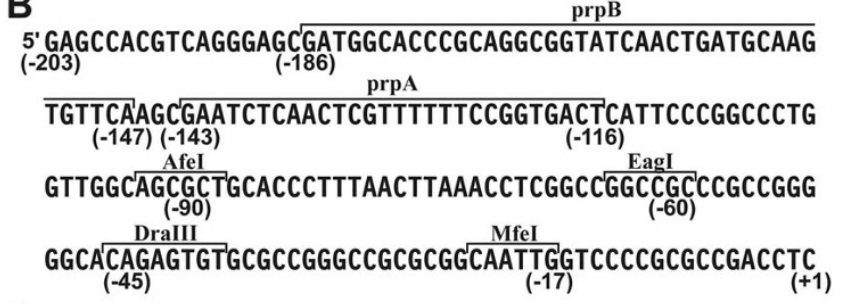

C

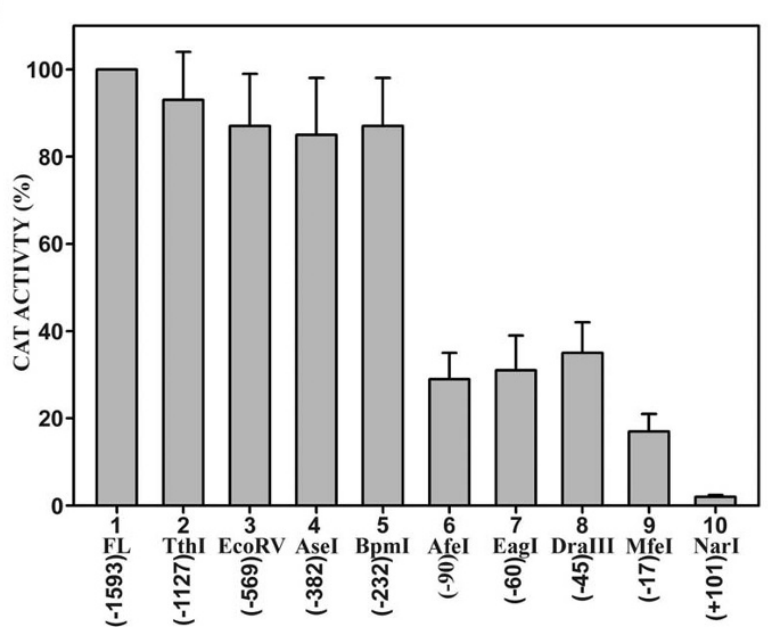

Figure I

Sequence of the PRNP promoter and expression analysis of 5' deletion constructs. (A) Schematic representation of the PRNP promoter from position - 1593 to +134 relative to the putative transcriptional start site $(+I)[2 I]$. The positions of relevant restriction sites used for deletion analysis are indicated. (B) Sequence of the proximal PRNP promoter from position -203 to + I. The positions of relevant restriction sites and nuclear factor binding domains PrpA and prpB are delineated by brackets. (C) PRPN promoter activities in HeLa cells as determined by transient transfection. The full-length (FL) promoter construct (column I) represents the sequence from position -1593 to +134 as indicated in A. The remaining 5' deletions (columns 2-10) were introduced at the restriction sites as shown in $A$ and B. CAT activities were normalized to identical $\beta$-galactosidase activities. The activity of each deletion was expressed relative to the activity of the full-length promoter construct (100\%). Each column shows the average value of 8 10 independent experiments with standard deviation.

ignated as bA1-bA3 (Fig. 3A, lanes 1-3). To determine binding specificity, mobility shift competition was carried out with a 20-fold excess of unlabeled oligonucleotides prpB and prpA. No binding competition was detected with unlabeled oligonucleotide prpB. In contrast, selfcompetition with unlabeled prpA eliminated labeled 


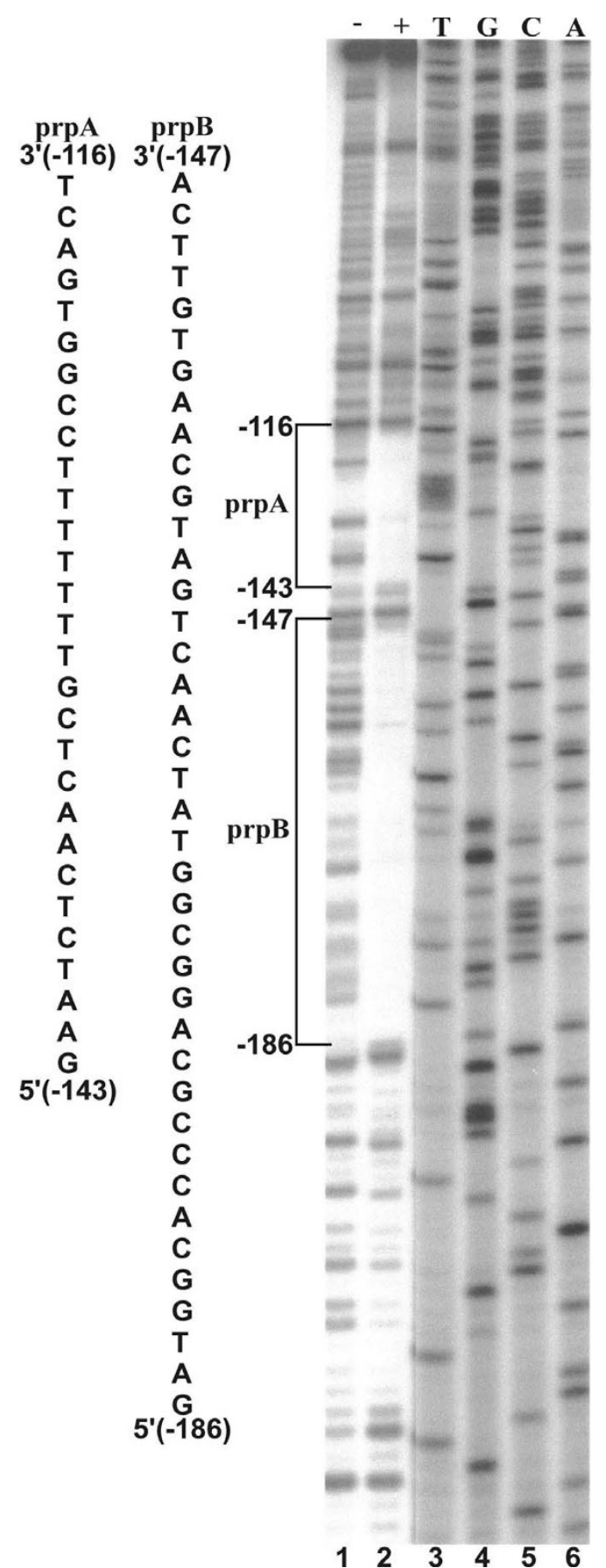

Figure 2

DNase I footprinting of a PRNP promoter fragment extending from position $\mathbf{- 2 5 9}$ to +2. partial DNase I digestion was carried out either with (lane 2 ) or without (lane I) HeLa cell nuclear extract. A dideoxy sequencing reaction with the same primer was included for positional reference (lanes 3-6). Two prominent footprints separated by three base pairs were designated prpB and prpA (brackets). The sequence of the DNase I protected domains are shown in the left panel. complex bA2, and partially competed complex bA1. Complex bA3 remained unaffected. This suggested that the factors that bound to the prpA region were distinct from those that bound to the prpB region and that complex bA2 and possibly complex bA1 represented sequence specific binding. Methylation interference was carried out to identify DNA contact points and to confirm the specificity of the binding complexes. Partial interference with the formation of complex bA2 occurred with three methylated $\mathrm{G}$ residue on the coding strand (Fig 3A, lanes 4-7) and two $G$ residues on the non-coding strand of oligonucleotide prpA (Fig 3A, lanes 8-10). Complexes bA1 and bA2 remained unaffected.

Mobility shift electrophoresis with labeled oligonucleotide prpB yielded five different binding complexes designated bB1-bB5. Of these complexes, bB1 and bB2 were exceedingly faint and complex bB3 was barely detectable. Mobility shift competition with excess unlabeled oligonucleotide prpA resulted in partial competition with complex bB4. The other complexes remained unaffected. Selfcompetition with oligonucleotide prpB completely competed labeled complex bB5 while the other complexes remained unaffected (Fig. 1B, lanes 1-3). This suggested that binding complex bB5 was sequence specific for the prpB region. Competition of prpA with complex bB4 indicated that this complex may share a binding motif common to both the prpA and $\mathrm{prpB}$ sequences. Partial methylation of domain prpB identified three $G$ residues on the coding strand with partial (Fig. 3B, lanes 4-9) and four $\mathrm{G}$ residues on the non-coding strand (Fig 3B, lanes 10-15) with complete binding interference for complex bB5. All other binding complexes were unaffected by Gmethylation.

The methylation interference experiments confirmed the results obtained by mobility shift electrophoresis. Binding complex bA2 on domain prpA and complex bB5 on prpB were sequence specific and they contained specific DNA contact points, defined by methylated $G$ residues that interfered with complex formation to the respective domains.

In other related studies, the DNA binding sites for adjacent transcriptional factors YY1 and E4BP4 were characterized on the ovine PRNP promoter [22]. Those binding sites are conserved between mammalian species and they are present on the human PRNP promoter between positions -290 to -258 . Consequently, they are located upstream from the prpA and prpB binding sites described here. PRNP expression was also found to be upregulated by SP1 and metal transcription factor-1 (MTF-1) [23]. However, the MTF-1 binding sites are located upstream (594) and downstream (-90) from the prpA and prpB binding sites. Furthermore, while SP1 consensus 
A

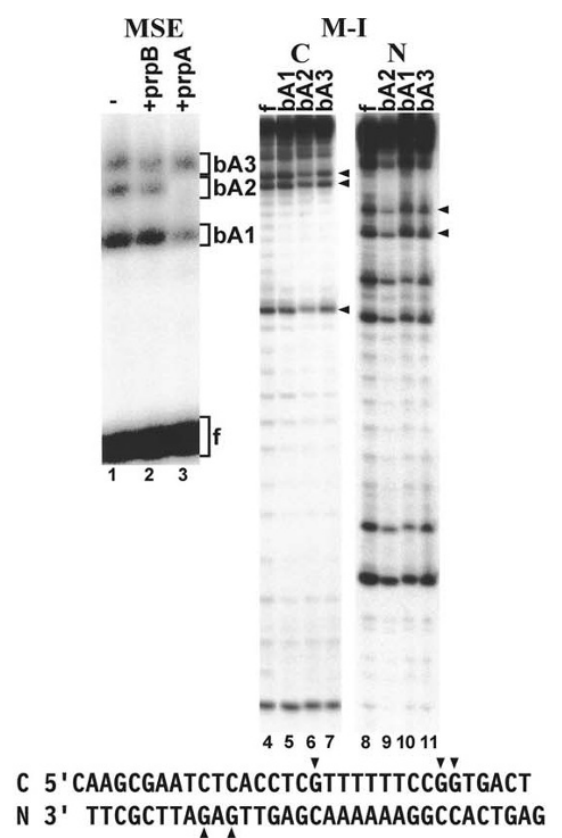

B

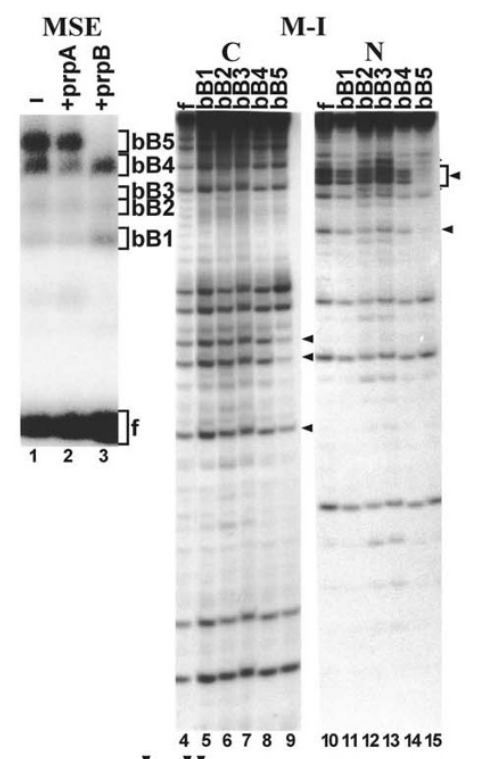

C 5 'GCGATGGCACC CǴCAG'GGGTATCAACTGATGCAAGTTTCA

N 3' GCTACCGTGGGCGTCGGCATAGTTGACTACGTTCAAAGTT

Figure 3

Mobility shift electrophoresis (MSE) and methylation interference (M-I) with 5 ' end labeled double stranded oligonucleotides encompassing the sequences of DNase I protected domains prpA and prpB. (A) MSE with 5' end labeled double stranded oligonucleotides encompassing the sequences of domain prpA (lane I, and lower panel). Brackets show binding complexes (bAI-bA3) and the free oligonucleotide (f). Mobility shift competition was performed with a 20-fold molar excess of unlabeled oligonucleotide prpB (lane2) and prpA (lane 3). Methylated G residues within domain prpA that partially interfere with binding of complex bA2 on the coding [(C), lanes 4-7] and noncoding [(N), lanes 8-II] strands are indicated by arrowheads. Lanes 4 and 8 show the methylation patterns of the free oligonucleotides. Lower panel shows the sequence of the double stranded oligonucleotide used and G-residues that interfere with binding to complex bA2. (B) Same as in A except that the sequence of DNase I protected domain PrpB was used. Mobility shift electrophoresis shows the formation of binding complexes bBI-bB5 with sequence-specific competition of complex bB5 (lanes I-3). The same binding complex also exhibits binding interference with indicated methylated $\mathrm{G}$ residues (bracket, arrowheads) on coding (lanes 4-9) and noncoding (lanes 10-15) strands. Lanes 4 and 10 show the methylation patterns of the free oligonucleotides. 
sequences are numerous throughout the PRNP promoter, they do not overlap with the prpA or prpB motifs. Binding domain prpB also overlaps with a consensus sequence for the NF-IL6 (C/EBP) transcriptional regulator [18]. However, Dlaska and Weiss [24] have presented a DNase I footprint of NF-IL6 binding to the iNOS promoter and that footprint has a different configuration from the one observed here. In addition, the methylated $\mathrm{G}$ residues that interfere with binding to $\mathrm{prpB}$ do not overlap with the NFIL6 consensus sequence. The 3' end of prpA also partially overlaps with a recognition sequence for transcription factor AP-1 [18]. However, binding of AP-1 to this domain is not supported by methylation interference results (Fig. 3). This suggests that the factors that bind to domains prpA and $\mathrm{prpB}$ are distinct from those previously described.

\section{Transfections with internal deletions of binding domains prpA and prpB}

To further investigate the function of binding domains prpA and prpB, the two domains were internally deleted within a full-length PRNP promoter construct. Domains prpA and prpB were either deleted separately (Fig 4, dA and $\mathrm{dB}$ ), or they were deleted together (Fig. 4, dAB). These internal deletions were then analyzed by transient trans-

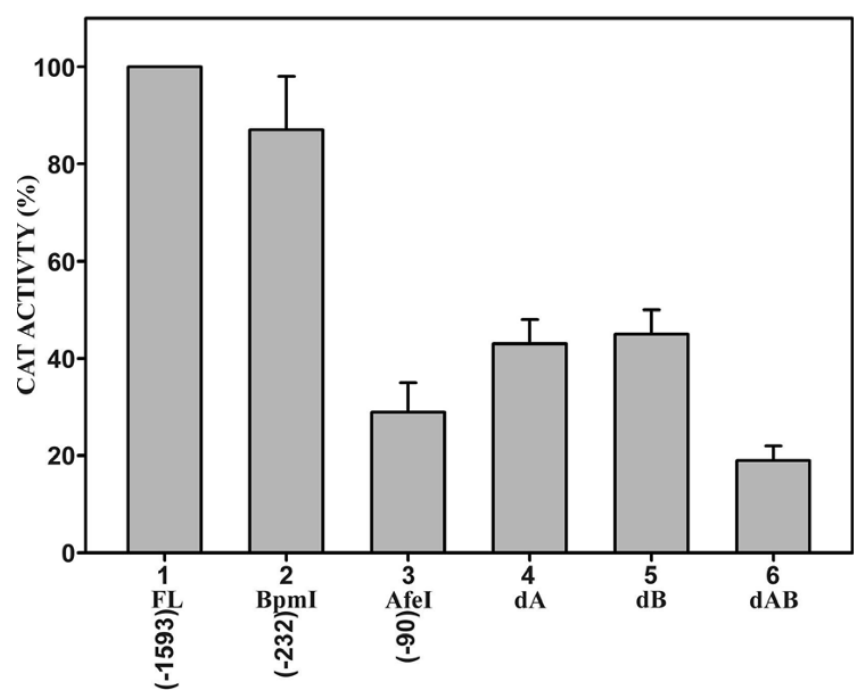

Figure 4

Activity of internal deletions within the full-length PRNP (-I593 to + I34) promoter construct (column I) in HeLa cells. Either binding domain prpA (column 4, dA) or prpB (column $5, \mathrm{~dB}$ ), or both domains (column $6, \mathrm{dAB}$ ) were internally deleted. Deletions from the $5^{\prime}$ end at restriction sites Bpm I (column 2, position -232) and Afe I (column 3 , position -90) are shown for comparison. The activity of each deletion was expressed relative to the activity of the full-length promoter construct (100\%). Each column shows the average of at least six independent experiments with standard deviation. fection in HeLa cells. Individually deleting binding domain prpA or prpB, reduced expression from the PRNP promoter by approximately the same amount to about $43 \%$ of the wild-type value (Fig. 4, FL). However, deleting both domains together had an additive effect and expression was reduced to less than $20 \%$ of the wild type value. The activity from the 5 ' deletion terminating at position 90 (Fig. 4, Afe I) was about 30\% of the full-length value. This construct is devoid of both binding domains prpA and $\mathrm{prpB}$, but for currently unknown reasons its level of activity is intermediate between the constructs containing individual internal deletions of prpA and prpB (43\%) and the combined deletion of both domains (18\%) [Fig. 4]. It is conceivable that additional negative regulatory motifs exist that are removed in the 5' deletion at position -90. Overall, these results suggest that binding domain prpA and prpB act in an additive manner to activate PRNP promoter expression.

\section{Conclusion}

A proximal promoter region extending from position -232 to +134 was identified that essentially confers full promoter activity in HeLa cells (Fig. 1). This region contains two nuclear factor-binding domains designated as prpA and prpB (Fig. 2). These domains are binding sites for distinct nuclear factors that are as yet unidentified (Fig. 3). The two domains independently activate expression from the PRNP promoter (Fig. 4). How these domains operate within context of the complete exon-intron structure of the endogenous PRNP gene or how they function within a neuronal cell background needs to be further investigated.

\section{Methods \\ Cell cultures, transfections, and enzyme assays}

HeLa cells (ATCC: CCL 2) were grown in DMEM supplemented with $5 \%$ fetal calf serum. Cells were transfected at $50 \%$ confluence with the ExGen 500 (Fermentas) transfection reagent according to the manufacturer's instructions. CAT and $\beta$-galactosidase assays were performed as described elsewhere [25]. CAT activities were quantitated with a BioRad GS-250 phosphor imager. The CAT activities resulting from the APP promoter constructs were normalized to corresponding $\beta$-galactosidase activities. Statistical significance (p-value) between selected final values was obtained with an unpaired t-test.

\section{Plasmids, promoter constructs, and oligonucleotides}

A promoter region of the human PRNP was obtained from HeLa cell genomic DNA by PCR with primers complementary to the chromosome 20 genomic sequence from position 4,605,433 to 4,607,158 [NCBI: NT_011387.8]. The resulting promoter fragment extended from the 3 ' end of exon $1(+134)$ to upstream position -1593 relative to a putative transcriptional start site $(+1)[21]$. Deletions from 
the 5'-end were generated by cutting with restriction enzymes at the indicated positions (Fig. 1A and 1B). All PRNP promoter constructs were analyzed in plasmid pCAT2bGAL [26]. This expression vector contains the bacterial genes CAT and LacZ. The LacZ gene is transcribed from the $\beta$-actin promoter and served as an internal control for experimental variations. The various PRNP promoter constructs were ligated into the mp18 polycloning site at the 5' end of the CAT gene. Internal deletions of the prpA and prpB domains were generated by in vitro mutagenesis [25,27]. Oligonucleotides were synthesized by Sigma Genosys or MWG Biotech and gel purified prior to use.

\section{Nuclear extracts and DNase I footprinting}

Nuclear extracts were prepared from HeLa cells grown in suspension to a density of $5-8 \times 10^{5}$ cells $/ \mathrm{ml}$ [25]. The final protein concentration in extracts was $10-15 \mathrm{mg} / \mathrm{ml}$ in buffer D containing $25 \mathrm{mM}$ Hepes, pH 7.6, $100 \mathrm{mM}$ $\mathrm{KCl}, 2 \mathrm{mM} \mathrm{MgCl}_{2}, 0.2 \mathrm{mM}$ EDTA, $1 \mathrm{mM}$ DTT, $0.1 \mathrm{mM}$ PMSF, and $10 \%$ glycerol. A promoter fragment extending from position -259 to +2 was amplified by PCR with a $\left[{ }^{32} \mathrm{P}\right]$ end-labeled forward primer complementary to the promoter sequence from position -259 to -235 and an unlabeled reverse primer complementary to the sequence from position -23 to +2 . The resulting DNA fragment was purified by agarose gel electrophoresis in $0.5 \times$ TBE. The labeled fragment $\left(1 \mu \mathrm{l}, 50 \times 10^{3} \mathrm{cpm}\right)$ was preincubated with $8 \mu \mathrm{l}$ of HeLa cell nuclear extract (+) or buffer D (-) in a total reaction volume of $33 \mu \mathrm{l}$ as described below for mobility shift electrophoresis. After 30 min of incubation, the fragment was partially digested with 10-60 units of DNase I (Roche) for $10 \mathrm{~min}$ at $25^{\circ} \mathrm{C}$. The reaction was stopped by adding $20 \mathrm{mM}$ EDTA and $0.5 \%$ SDS, followed by phenol/chloroform extraction and ethanol precipitation. The dried pellet was solubilized in formamide loading buffer and electrophoresed in a $6 \%$ sequencing gel.

\section{Mobility shift electrophoresis and methylation interference}

Double-stranded oligonucleotides comprising the sequences containing nuclear factor binding domains prpA or prpB (Fig. 3) were 5 ' end-labeled with $\left[\gamma^{32} \mathrm{P}\right]$ ATP [28]. Binding reactions were assembled with $5 \mu \mathrm{l}$ of nuclear extract, $1 \mu \mathrm{l}\left(50 \times 10^{3} \mathrm{cpm}\right)$ of labeled oligonucleotide and $24 \mu \mathrm{l}$ of buffer containing $40 \mathrm{mM}$ Hepes pH 7.6, $2 \mathrm{mM} \mathrm{MgCl}_{2}, 0.1 \mathrm{mM}$ EDTA, $1 \mathrm{mM}$ DTT, $50 \mathrm{mM} \mathrm{KCl}$, $10 \%$ glycerol, supplemented with $2 \mu \mathrm{g}$ of poly(dI-dC)poly(dI-dC) [GE Healthcare]. After 30 min incubation at $25^{\circ} \mathrm{C}$, the binding reaction products were separated by electrophoresis in $1 \%$ agarose gels containing $0.5 \times$ TBE at $150 \mathrm{~V}$ constant voltage. The gels were dried and analyzed with a BioRad GS-250 Phosphor Imager. In competition assays, unlabeled oligonucleotides were premixed with labeled oligonucleotides at a 20-fold molar excess before adding them to the binding reaction.

Oligonucleotides comprising the sequences of binding domains prpA and prpB were partially methylated and 5 ' end-labeled on either the coding or non-coding strands as described [28]. After mobility shift electrophoresis, the binding complexes (Fig. 3) and the free oligonucleotide were isolated, cleaved with piperidine and separated on a $20 \%$ sequencing gel.

\section{List of abbreviations used}

TBE: Tris-borate-EDTA; PMSF: phenylmethylsulfonyl fluoride; DMEM: Dulbecco's modified Eagle's medium; PRNP: prion protein gene; PrP: prion protein; ORF-open reading frame; TSE: transmissible spongiform encephalopathy; PCR: polymerase chain reaction; ATCC: American Type Culture Collection; CAT: chloramphenicol acetyltransferase; NGF: nerve growth factor.

\section{Competing interests}

The authors declare that they have no competing interests.

\section{Authors' contributions}

MT, WQ, and NI carried out molecular cloning and transfections. MT, NI, and AV performed DNase I footprinting and mobility shift electrophoresis. WQ and MT participated in methylation interference and in vitro mutagenesis experiments. All authors contributed to data analysis and WQ wrote the manuscript. All authors read and approved the final manuscript.

\section{Acknowledgements}

This work was supported by National Institutes of Health grant NS30994.

\section{References}

I. Prusiner SB: Prions. Proc Natl Acad Sci USA 1998, 95: I3363-13383.

2. Brandner S, Isenmann S, Raeber A, Fischer M, Sailer A, Kobayashi $Y$, Marino S, Weissmann C, Aguzzi A: Normal host prion protein necessary for scrapie-induced neurotoxicity. Nature 1996, 379:339-343.

3. Büeler H, Aguzzi A, Sailer A, Greiner RA, Autenried P, Aguet M, Weissmann C: Mice devoid of PrP are resistant to scrapie. Cell 1993, 73:1339-1347.

4. Westaway D, Mirenda CA, Foster D, Zebarjadian Y, Scott M, Torchia M, Yang SL, Serban H, DeArmond Sj, Ebeling C, Prusiner SB, Carlson GA: Paradoxical shortening of scrapie incubation times by expression of prion protein transgenes derived from long incubation period mice. Neuron 1991, 7:59-68.

5. Lee IY, Westaway D, Smit AFA, Wang K, Seto J, Chen L, Acharya C Ankener M, Baskin D, Cooper C, Yao H, Prusiner SB, Hood LE: Complete genomic sequence and analysis of the prion protein gene region from three mammalian species. Genome Res 1998, 8: $1022-1037$.

6. Oesch B, Westaway D, Wälchli M, Mc Kinley MP, Kent SB, Aebersold R, Barry RA, Tempst P, Teplow DG, Hood LE, Prusiner SB, Weissman C: A cellular gene encodes scrapie PrP 27-30 protein. Cell 1985, 40:735-746.

7. Manson J, West JD, Thomson V, McBride P, Kaufman MH, Hope J: The prion protein gene: a role in mouse embryogenesis? Development 1992, II 5:117-122.

8. Varela-Nallar L, Toledo EM, Larrondo LF, Cabral AL, Martins VR, Inestrosa NC: Induction of cellular prion protein gene expres- 
sion by copper in neurons. Am J Physiol Cell Physiol 2006, 290:C27I-28I.

9. Mobley WC, Neve RL, Prusiner SB, McKinley MP: Nerve growth factor increases mRNA levels for the prion protein and the $\beta$-amyloid protein precursor in developing hamster brain. Proc Natl Acad Sci USA 1988, 85:98I I-98I5.

10. Shyu WC, Chen CP, Saeki K, Kubosaki A, Matusmoto Y, Onodera T, Ding DC, Chiang MF, Lee YJ, Lin SZ, Li H: Hypoglycemia enhances the expression of prion protein and heat-shock protein $\mathbf{7 0}$ in a mouse neuroblastoma cell line. J Neurosci Res 2005, 80:887-894.

II. Zawlik I, Witusik M, Hulas-Bigoszewska K, Piaskowski S, Szybka M, Golanska E, Liberski PP, Rieske P: Regulation of PrPC expression: nerve growth factor (NGF) activates the prion gene promoter through the MEKI pathway in PCI 2 cells. Neurosci Lett 2006, 400:58-62.

12. Liang J, Bai F, Luo G, Wang J, Liu J, Ge F, Pan Y, Yao L, Du R, Li X, Fan $\mathrm{R}$, Zhang $\mathrm{H}$, Guo X, Wu K, Fan D: Hypoxia induced overexpression of $\operatorname{PrP}(C)$ in gastric cancer cell lines. Cancer Biol Ther 2007, 6:769-774.

13. Saeki K, Matsumoto $Y$, Matsumoto $Y$, Onodera T: Identification of a promoter region in the rat prion protein gene. Biochem Biophys Res Commun 1996, 219:47-52.

14. Inoue S, Tanaka M, Horiuchi M, Ishiguro N, Shinagawa M: Characterization of the bovine prion protein gene: the expression requires interaction between the promoter and intron. J Vet Med Sci 1997, 59:175-183.

15. Baybutt H, Manson J: Characterisation of two promoters for prion protein (PrP) gene expression in neuronal cells. Gene 1997, I 84: I25-|3|.

16. Premzl M, Delbridge M, Gready JE, Wilson P, Johnson M, Davis J, Kuczek E, Marshall Graves JA: The prion protein gene: identifying regulatory signals using marsupial sequence. Gene 2005, 349: $|2|-\mid 34$.

17. Funke-Kaiser $\mathrm{H}$, Theis $\mathrm{S}$, Behrouzi T, Thomas A, Scheuch K, Zollmann FS, Paterka M, Paul M, Orzechowski HD: Functional characterization of the human prion protein promoter in neuronal and endothelial cells. J Mol Med 200I, 79:529-535.

18. Mahal SP, Asante EA, Antoniou M, Collinge J: Isolation and functional characterisation of the promoter region of the human prion protein gene. Gene 200I, 268:I05-II4.

19. Mead S, Mahal SP, Beck J, Campbell T, Farrall M, Fisher E, Collinge J: Sporadic-but not variant-Creutzfeldt-Jakob disease is associated with polymorphisms upstream of PRNP exon I. Am J Hum Genet 200I, 69:1225-1235.

20. Sander P, Hamann H, Drögemüller C, Kashkevich K, Schiebel K, Leeb $\mathrm{T}$ : Bovine prion protein gene (PRNP) promoter polymorphisms modulate PRNP expression and may be responsible for differences in bovine spongiform encephalopathy susceptibility. I Biol Chem 2005, 280:37408-374I4.

21. Puckett C, Concannon P, Casey C, Hood L: Genomic structure of the human prion protein gene. Am J Hum Genet 1991, 49:320-329.

22. Burgess STG, Shen C, Ferguson LA, O'Neill GT, Docherty K, Hunter $\mathrm{N}$, Goldmann W: Identification of adjacent binding sites for the YYI and E4BP4 transcription factors in the ovine PrP (Prion) gene promoter. J Biol Chem 2009, 284:6716-6724.

23. Bellingham SA, Coleman LA, Masters CL, Camakaris J, Hill AF: Regulation of prion gene expression by transcription factors SPI and metal transcription factor-I. J Biol Chem 2009:129I-I30I.

24. Dlaska M, Weiss G: Central role of transcription factor NF-IL6 for cytokine and iron-mediated regulation of murine inducible nitric oxide synthase expression. J Immunol 1999 162:617|-6177.

25. Quitschke WW, Matthews JP, Kraus RJ, Vostrov AA: The initiator element and proximal upstream sequences affect transcriptional activity and start site selection in the amyloid $\beta$-protein precursor promoter. J Biol Chem 1996, 27 I:2223I-22239.

26. Quitschke WW, Goldgaber D: The amyloid $\beta$-protein precursor promoter, A region essential for transcriptional activity contains a nuclear factor binding domain. J Biol Chem 1992, 267: 17362-17368

27. Kunkel TA: Rapid and efficient site-specific mutagenesis without phenotypic selection. Proc Natl Acad Sci USA 1985, 82:488-492.
28. Quitschke WW: Two nuclear factor binding domains activate expression from the human amyloid $\beta$-protein precursor promoter. J Biol Chem 1994, 269:2 I229-21233.
Publish with Bio Med Central and every scientist can read your work free of charge

"BioMed Central will be the most significant development for disseminating the results of biomedical research in our lifetime. "

Sir Paul Nurse, Cancer Research UK

Your research papers will be:

- available free of charge to the entire biomedical community

- peer reviewed and published immediately upon acceptance

- cited in PubMed and archived on PubMed Central

- yours - you keep the copyright 